\title{
Modular Plug-in High Performance Integrated Single-Phase Inverter
}

\author{
Adane Kassa Solomon, Alberto Castellazzi \\ University of Nottingham, PEMC Group \\ University Park, NG7 2RD \\ Adane_Kassa.Solomon@nottingham.ac.uk \\ Alberto.Castellazzi@nottingham.ac.uk
}

\begin{abstract}
The recent research exercises have targeted the transfer of the sandwich package benefits to application bespoke switch design, including flip-chip and device stacking topology [1]. This work presents the development of a highly integrated power switch, based on $70 \mu \mathrm{m}$ thin IGBTs and diodes rated at $600 \mathrm{~V} / 200 \mathrm{~A}$ along with customized connectors to interface quickly the gate driver, the input, and the load side PCBs. This modular system has been designed with the aim to achieve high performance including the modularity and maintainability of power converter that can be worse in a system integrated into a single package.
\end{abstract}

\section{Introduction}

This paper presents the reliable integration of a Halfbridge switch based on latest generation 70um thin devices rated at $600 \mathrm{~V}-200 \mathrm{~A}$ IGBTs and diodes. The use of thin devices in the switch implementation offers not only superior electrical but also thermal performance, as compared with thicker ones, and brings along increased power density figures [2]. In particular, it advances the state-of-the-art as regards a recently presented packaging concept, which aims the optimisation from the application point of view [3]. As pointed out in [4] and illustrated in Fig. 1 for improving switching performance, packaging and integration design should aim at integrating the positive and negative switching cells, that is the high-side transistor/low-side diode and the low-side transistor/highside diode pairs respectively.

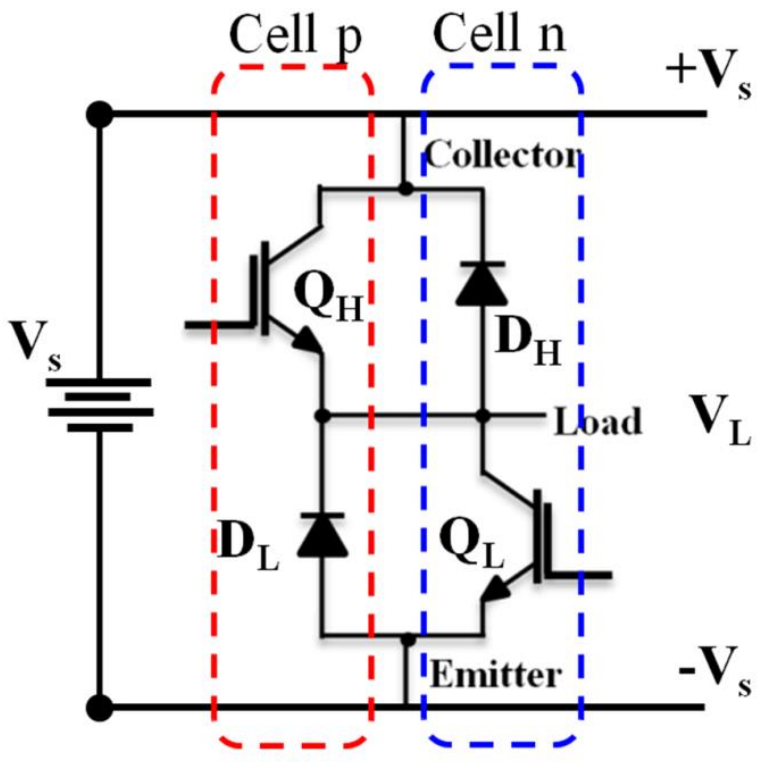

Fig.1 Half-bridge switch (HBS) schematic diagram
Based on the above considerations, this paper addresses an advanced integration approach of a Half-bridge power switch. The paper particularly is trying to optimize the switching and thermal performance of the HBS, and design a double-etched Si3N4 substrate based HBS along with plug-in connectors on either side of the switch where the power and drive loops kept very small. Moreover, the integration approach enables to easily keep separate driving and power loops, positioning the relative electrical interconnections on opposite sides of the switch; the approach is also suitable for double-sided (liquid) cooling of the devices.

\section{Half-bridge switch Model and simulation}

The developed switch model was characterized electromagnetically, employing structural numerical analysis tools in [5,6]. A preliminary Finite element (FE) thermal modelling and simulation for the HBS has been carried out to compare the maximum juncture temperature of the chips at different environmental conditions using Silicon-Nitrate substrate. The modeling and simulation were done using commercial finite element analysis software ABAQUS 6.10-2 and its graphic user interface CAE [7]. Here, both the top and bottom substrates are active metal brazed (AMB) consisting of $0.635 \mathrm{~mm}$ thick silicon nitride substrate sandwiched by $0.2 \mathrm{~mm}$ thick copper on both sides etched with $0.2 \mathrm{~mm}$ thickness of copper bump properly aligned to the corresponding small interconnection pads of the chips (e.g. Emitter, gate) [8]. As thermal load, a total power dissipation of $300 \mathrm{~W}$ is considered by the semiconductor devices where a $100 \mathrm{~W}$ and 50W of power are generated inside each IGBTs and Diodes respectively.

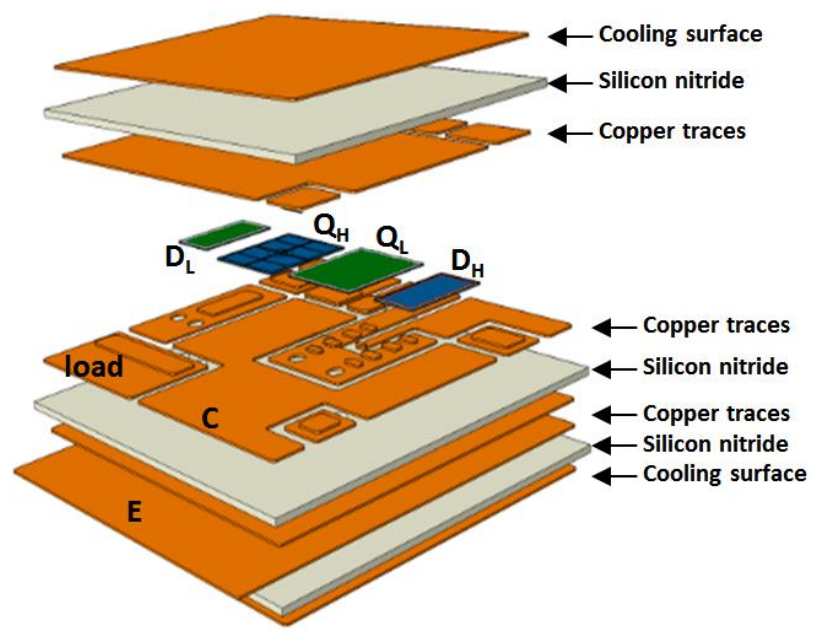

Fig.2 Exploded version of the 3D model HBS 
Both top and bottom cooling surfaces were subjected to heat-transfer coefficient of " $\mathrm{h}=5000 \mathrm{~W} / \mathrm{m}^{2} \mathrm{~K}$ ", to account for the contributions of a typical forced water cooling system in power electronics as depicted in Fig.3. The heat transfer coefficient of all the other surfaces was assumed to be zero (adiabatic condition).

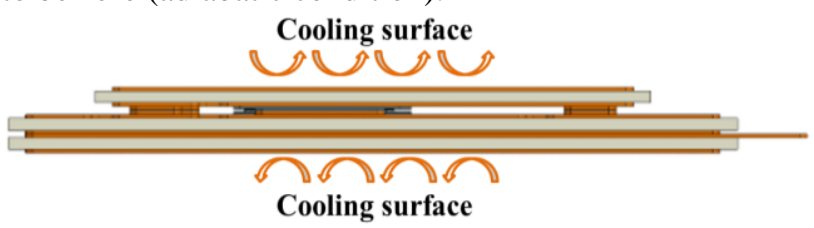

Fig.3 Boundary condition of heat exchange

The joule heating created by the presence of low resistance of the copper traces is considered to be neglected at this stage. The thermal properties of the materials listed in Table 1 were taken from [1]:

\begin{tabular}{|lc|}
\hline Material & Thermal conductivity \\
\hline Copper & $398 \mathrm{~W} / \mathrm{mK}$ \\
\hline Silicon & $146 \mathrm{~W} / \mathrm{mK}$ \\
\hline Silicon nitride & $70 \mathrm{~W} / \mathrm{mK}$ \\
\hline Aluminium & $238 \mathrm{~W} / \mathrm{mK}$ \\
\hline Solder $($ SnAg) & $33 \mathrm{~W} / \mathrm{mK}$ \\
\hline
\end{tabular}

Table 1Material thermal properties

Three environmental conditions were considered where the assembly is subjected to ambient $(293 \mathrm{~K})$, worst (318K, e.g. commercial application) and harsh (348K, e.g. avionic) temperatures. The maximum allowable temperature of IGBT, which cannot be exceeded in any circumstances, is $175^{\circ} \mathrm{C}$ (448K) [9]. Fig.4 shows the contour of thermal map for the ambient temperature condition and the result is clearly well below the maximum junction temperature requirement.

NT11

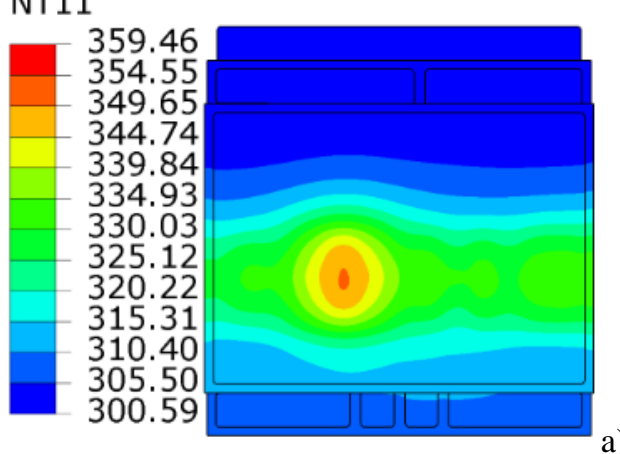

NT11

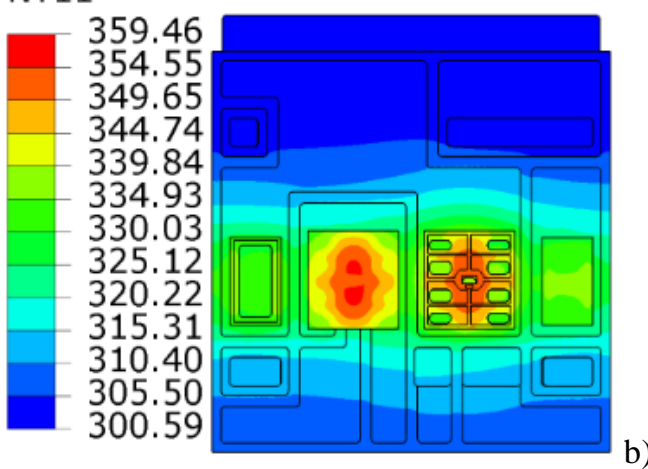

Fig.4 Steady state thermal simulation result, Tamb=293K; a) full assembly and b) assembly without top substrate
The simulation results illustrate that in the worst and harsh environment, the maximum junction temperature is $384 \mathrm{~K}$ and $414 \mathrm{~K}$ respectively. However, it is still below the target maximum junction temperature.

\section{Half-bridge switch system assembly}

The HBS prototype shown in Fig.6 was constructed with the double etched Si3N4 (silicon nitride) Kyocera substrate and the detailed construction is presented in [5]. The schematic of the overall system is depicted in Fig.7 showing the possible new solutions to the end connectors of the HBS; the gate-driver connector, the HBS module, and the power connector.

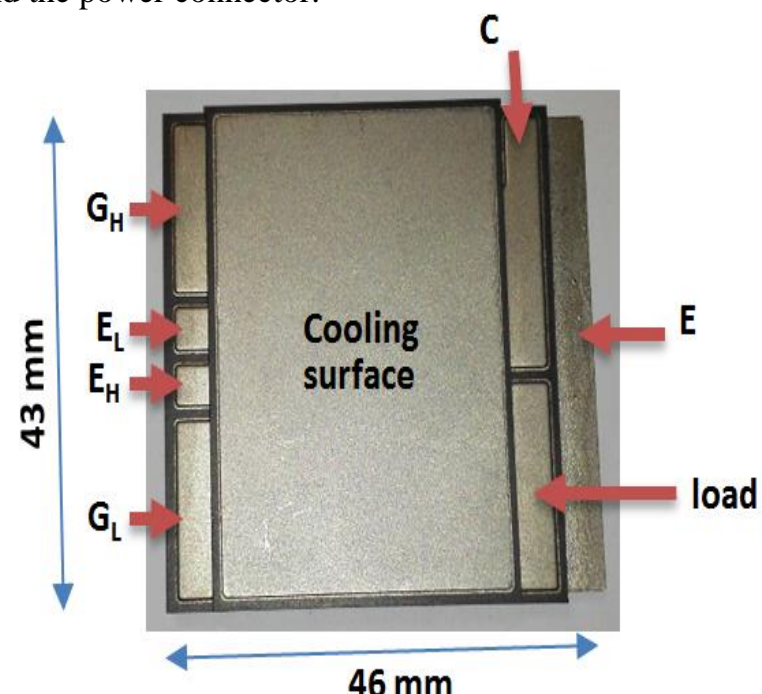

Fig.6 HBS assembly prototype

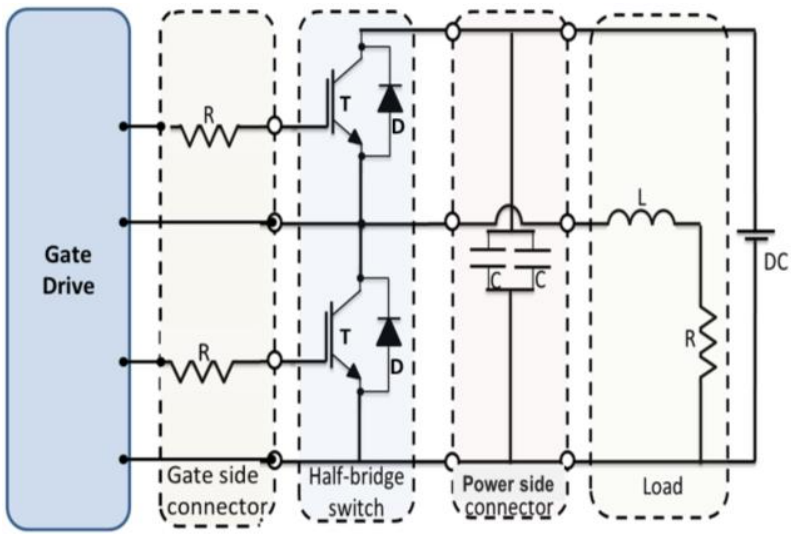

Fig.7 Full system and experimental setup diagram

In the connectors some ceramic capacitors have been embedded, with a capacitance suitable to filter switching ringing. The idea was to design not only a new highly integrated sandwich half-bridge module, but also new connectors to keep the lengths of the connections as short as possible in order to have small stray inductances, both for gate and power loops. Two connectors are designed to connect the gate drive and load side of the assembly in order to have better accessibility and modularity. High frequency capacitors are embedded into the load side connectors, as well as in the gate drive side; the gate-driver connector is designed to embed some coupling capacitors and gate resistors. Fig.8 illustrates the power side connector details which contain properly designed copper 
plates for emitter, collector and load with insulating and housing of plastic.

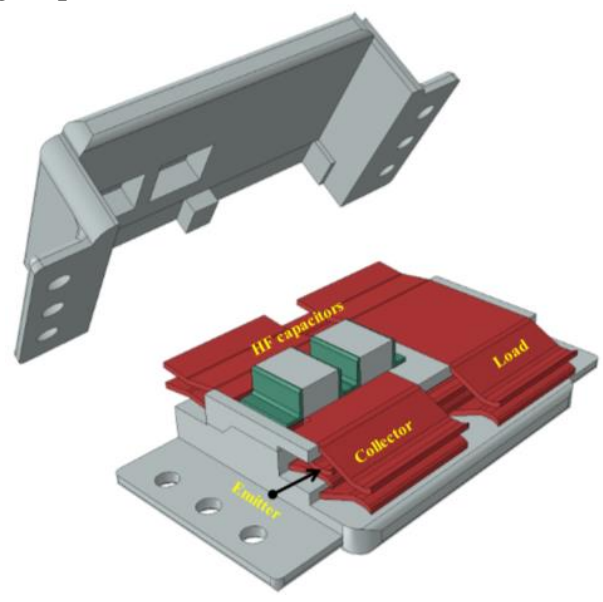

Fig.8 Power side connector

Fig. 8 shows the control side connector details which contain properly designed copper plates for emitter, gate and supporter with insulating and housing of plastic. Two $10 \Omega$ resistors are also embedded inside the connector.

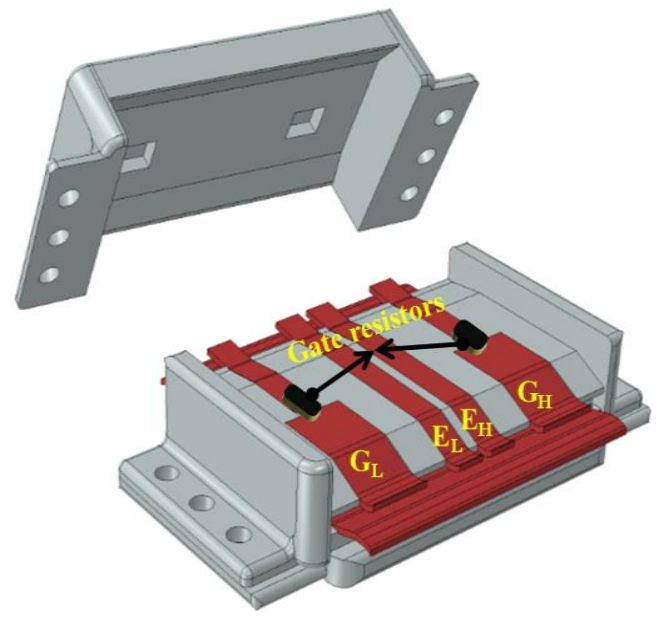

Fig.9 Gate side connector

A first version of the water cooling system was designed and the water flowing path inside the power module is planned to directly target the hot spot (rectangular open area shown in Fig.10), where the power devices are located. The liquid flows from the inlet toward the top cooling surface, and once it passes this surface, it turns down to flow through the bottom substrate of the switch to get to the outlet. The overall cooling system is hermetically assembled and fixed with screws using rectangular gaskets (see Fig.10) designed to seal the plastic and the switch cooling surface together.

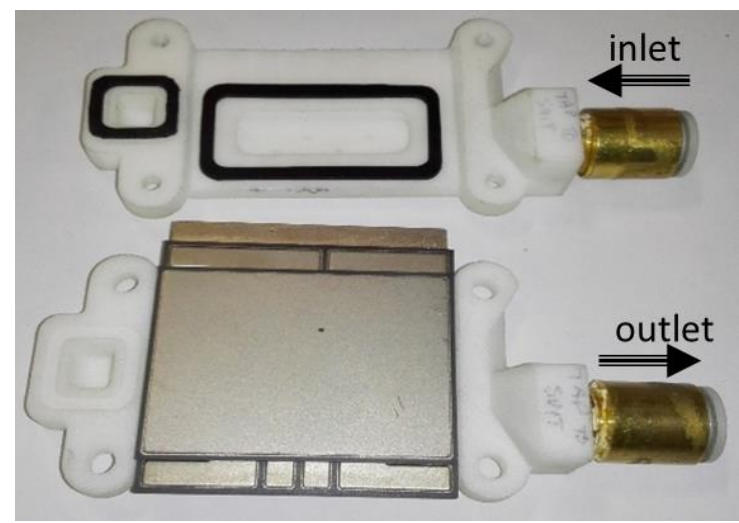

Fig. 10 cooling system

Fig. 11 shows the prototype of a single phase inverter made mainly to test the performances of the HBS and the quick connectors. The idea here is to produce a high reliable plug-in system to reduce downtime and drive down maintenance costs of repairable systems. Waveforms of the collector-emitter voltage measured during early electrical tests have shown extremely clean switching transitions, confirming a very low parasitic inductance of the power loop [6].

\section{Experimental test}

Here, the gate drive and power PCB boards are kept very close to the switch to minimize the noise pick up at the gate signals and parasitic inductances. As this power module is designed with DC-link capacitor; it requires large capacitance values to minimize the low frequency voltage oscillation at the capacitor. Both electrolytic and high frequency capacitors are used to suppress the voltage oscillations at the DC bus [10].

A preliminary functional test was carried out in a basic half-bridge dc-ac converter at an input DC voltage of $300 \mathrm{~V}, 30 \Omega$ load resistor, and $8.4 \mathrm{mH}$ load inductor. Fig. 11 also indicates the assembled hardware and test setup, corresponding to the circuit schematic of Fig.7.

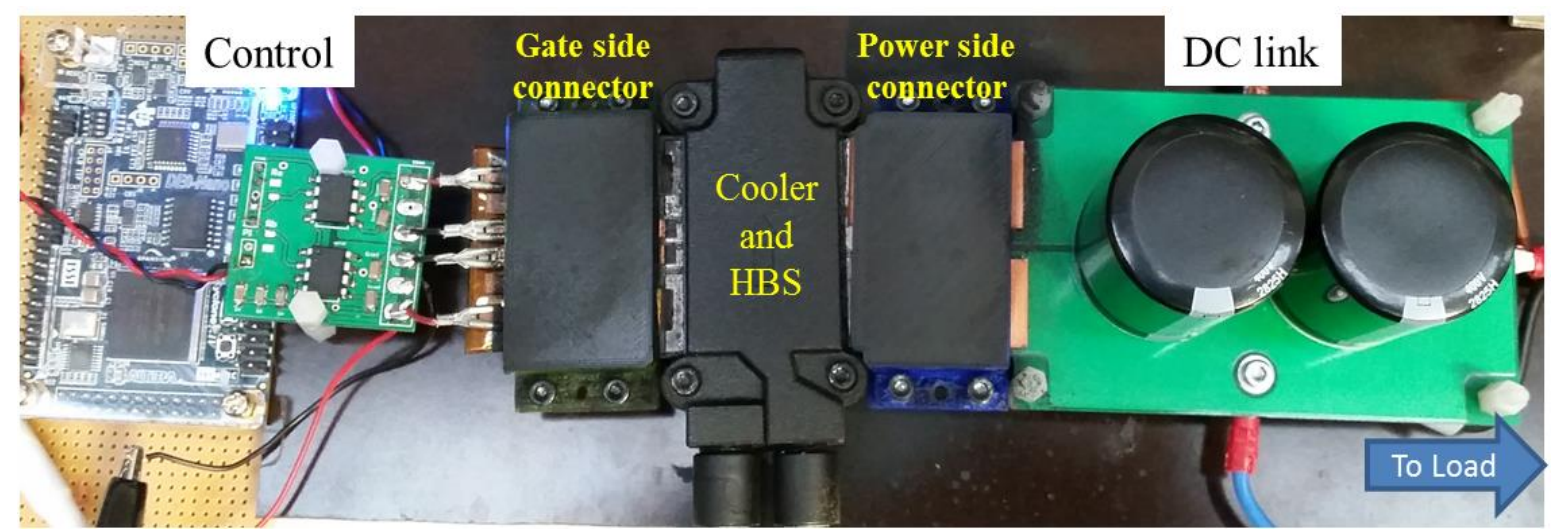

Fig.11 Single phase inverter experimental setup 
In this set-up, a dedicated test board was built, which accommodates passives and interface with the control platform where a fixed dead time of $0.5 \mu$ s was chosen, and the inverter is tested in an open-loop system. The cooling liquid temperature was regulated to $25 \mathrm{C}$ and a $40 \%$ less toxic propylene glycol was used for corrosion and frost protection.

The representative experimental results shown in Fig.13 are the current through the load and the voltage across the top switch having nearly total absence of voltage overshoot during the turn off transition period of the switch. Thermal map reading on the surface of the cooler shown in Fig.13a and 13b are experimentally captured result using infrared camera when the system is operating at the voltage stated above and the simulation contour respectively [11].
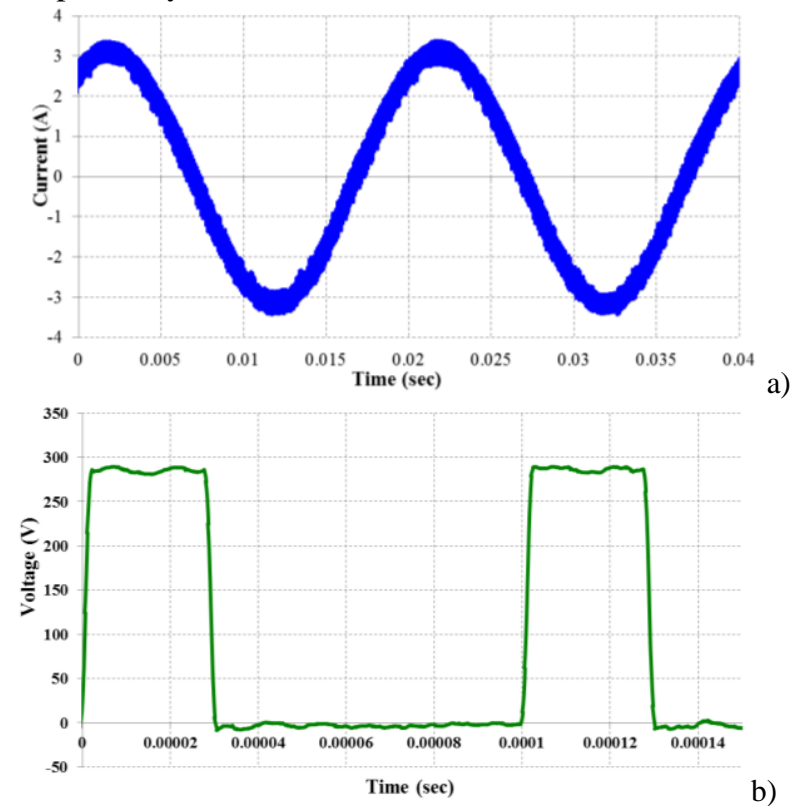

Fig.12 Representative experimental waveforms; a) output current and b) switch voltage
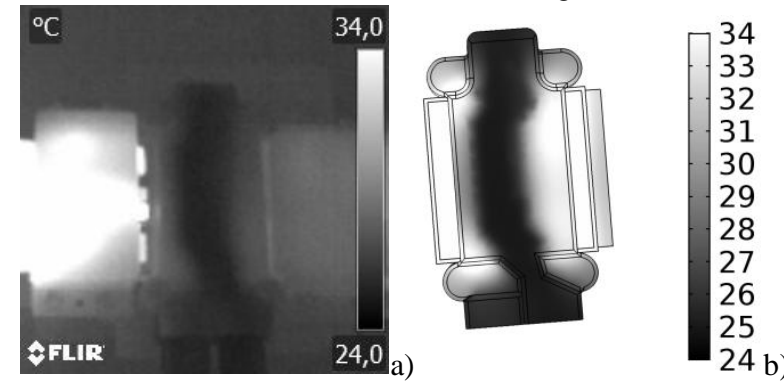

Fig.13 Thermal plot on the upper surface of the cooler with HBS assembly [11]; a) Experimental b) Simulation

\section{Conclusion and outlook}

This paper presents an innovative integration scheme for power switches which brings along newly plug-in assembly solutions. The plug-in feature can support in reducing maintenance in repairable systems without losing the high-performance capability. A single-phase inverter prototype with a new double-sided liquid cooled bond wire-less sandwich based HBS together with new doubleedge connectors having integrated capacitors and resistors have been presented. The basic thermal simulation results show the ability to perform well even in the harsh environmental conditions. The functionality of the whole system has been demonstrated in preliminary test. These preliminary experimental results confirm the perfect functionality of the switch and, more importantly, reveal a very contained value of parasitic inductance. In future it is anticipated to work on the mechanical behavior of the connectors and insulation of the switches for dielectric strength and protection from pollution which is the stringent requirement for high voltage operation and technology transfer.

\section{References}

[1] J.F. Li, A. Castellazzi, A. Solomon, C.M. Johnson, "Reliable Integration of Double-Sided Cooled Stacked Power Switches based on $70 \mu \mathrm{m}$ Thin IGBTs and Diodes," CIPS2012, March 2012, Nuremberg, Germany.

[2] H. R. Chang, J. Bu, G. Kong, and R. Labayen, "300 A $650 \mathrm{~V} 70$ um thin IGBTs with double-sided cooling," in Proc. IEEE ISPSD ICs, San Diego, CA, USA, 2011, pp. 320-323.

[3] X. S. Liu, S. Haque, and G.-Q. Lu, "Three-dimensional flip-chip on flex packaging for power electronics applications," IEEE Trans. Adv. Packag.,vol. 24, no. 1, pp. 1-9, Feb. 2001.

[4]F.Z. Peng, "Revisit Power Conversion Circuit Topologies-Recent Advances and Applications", in Proc. of the 6th IEEE International Power Electronics and Motion Control Conference (IPEMC-ECCE Asia 2009), Wuhan, China, May 2009.

[5] Adane K. Solomon, A. Castellazzi, "Alternative integration scheme for half-bridge switch using double etched Si3N4 substrate" in Proceedings of the International Conferenceon ESTC 2014.

[6] A. K. Solomon, A. Castellazzi, N. Delmonte, P. Cova, "Modular power Converter Integration Based On NonConventional Power Switch Assembly and Interconnects", Proc. 9th International Conference on Power Electronics, ICPE 2015-ECCE ASIA, Seoul, Korea, Jun. 1-5, 2015, pp. 837-843.

[7]Abaqus unified FEA, Complete solutions for realistic simulation. [Online]. Available: http://www.3ds.com

[8]Kyocera corporation [Online]. Available: http://global.kyocera.com/

[9] Infineon Technologies AG [Online]. Available: https://www.infineon.com/

[10]EPCOS; TDK Group Company [online] http://en.tdk.eu/inf/20/10/ds/B58031I5105M002 LP.

[11]P. Cova , N. Delmonte, A.K. Solomon, A. Castellazz; "Thermal design optimization of novel modular power converter assembly enabling higher performance, reliability and availability" Accepted at 27 th European Symposium on Reliability of Electronic Devices, Failure physiscs and analysis (ESREF2016). 